\title{
A randomized controlled trial of a mindfulness- based intervention program for people with schizophrenia: 6-month follow-up
}

This article was published in the following Dove Press journal:

Neuropsychiatric Disease and Treatment

7 December 2016

Number of times this article has been viewed

\author{
Li-Qun Wang' \\ Wai Tong Chien ${ }^{2}$ \\ Lai King Yip ${ }^{2}$ \\ Thanos Karatzias ${ }^{3}$ \\ 'School of Nursing, Jilin Medical \\ College, Jilin, ${ }^{2}$ School of Nursing, \\ The Hong Kong Polytechnic University, \\ Hung Hom, Kowloon, Hong Kong \\ SAR, People's Republic of China; \\ ${ }^{3}$ Faculty of Health, Life and Social \\ Sciences, Edinburgh Napier University, \\ Edinburgh, Scotland, UK
}

\begin{abstract}
Mindfulness-based interventions have been increasingly evidenced to be effective in different mental illnesses but limited in schizophrenia. This single-blind, multisite randomized controlled trial tested the effects of a mindfulness-based psychoeducation group program (MPGP in addition to usual care) versus a conventional psychoeducation group program (CPGP) versus treatment-as-usual (TAU) alone, in schizophrenia spectrum disorders over a 6-month follow-up. In each of the two study sites (outpatient clinics), 69 outpatients with schizophrenia or its subtypes $(\mathrm{N}=138)$ were randomly allocated to one of the three study groups $(n=46)$ after baseline measurements and underwent 6 months of intervention. Primary outcomes including patients' mental state and rehospitalization rate and other secondary outcomes were assessed at entry and at 1 week and 6 months. One hundred and thirty-one (95\%) participants completed the interventions assigned and one to two post-tests. Multivariate analyses of variance (followed by univariate contrast tests) indicated that the MPGP participants reported greater reductions in their psychotic symptoms $(P=0.003)$ and length/duration of rehospitalizations $(P=0.005)$ at 6-month follow-up. Patients in the MPGP group also reported greater improvements in their insight into illness/treatment $(P=0.0008)$ and level of functioning $(P=0.002)$ than the CPGP and TAU alone at the 1-week and 6-month follow-up. Overall, the findings suggest that MPGP can be useful in improving the short- to medium-term clinical outcomes of outpatients with schizophrenia spectrum disorders, not only in terms of their mental state and risk of relapse but also their insight into illness/treatment and psychosocial functioning.
\end{abstract}

Keywords: mindfulness intervention, psychoeducation, functioning, insight into illness, psychotic symptoms, rehospitalization, schizophrenia

\section{Introduction}

Schizophrenia patients, constituting more than half of those with severe mental illness in global communities, ${ }^{1}$ are characterized by profound disturbances and worsening in their cognition, emotion, and psychosocial functioning. ${ }^{2}$ These patients often experience high relapses and demands for mental health services. While there are increasing advanced antipsychotic medications to reduce the psychotic symptoms, many patients still experience disabling residual symptoms and impaired functioning. Different approaches to psychosocial interventions provide significant benefits in improving patients' symptoms, drug compliance, and relapse, but inconsistent and inconclusive results/effects in other psychosocial health conditions. ${ }^{1,3}$

As recommended by a recent guideline of the National Institute for Health and Clinical Excellence in the $\mathrm{UK},{ }^{2}$ core interventions for early stage (eg, $<5$ years) or acute schizophrenia should include psychoeducation, medication management, and
Correspondence: Wai Tong Chien School of Nursing, The Hong Kong Polytechnic University, Hung Hom, Kowloon, Hong Kong SAR, People's Republic of China

Tel +852 27665648

Fax +852 2334 I I 24

Email wai.tong.chien@polyu.edu.hk 
family support. They should also include those aiming to improve their insight into and self-management of the illness. However, most current approaches to psychosocial intervention have less attention in patients' acceptance of schizophrenia and incomprehensible and stressful experiences of the psychotic symptoms. ${ }^{4,5}$ Many of them may also contain limited strategies in empowering self-management of the illness. ${ }^{1,4}$ It has also increasingly been recognized that patients would be unable, inconvenient, or disempowered to participate in lengthy (at least 6-9 months) and knowledge/conceptual heavy-loaded psychoeducation, or other psychosocial intervention programs. ${ }^{6,7}$ With a differential understanding of schizophrenia and insight into its treatment needed, these patients can better cope with their psychotic symptoms and related behaviors and subsequently improve their treatment adherence and other longer-term patient outcomes. ${ }^{8}$

Recent controlled trials suggested that the standardized mindfulness-based stress reduction (MBSR) or mindfulnessbased cognitive intervention programs are evidenced to empower patients' self-care and symptom management, insight into the illness, and accepting and overcoming with distressing thoughts in depressive and anxious conditions. ${ }^{9,10}$ Both approaches are found to be effective treatments of a wide variety of chronic physical and mental health problems. ${ }^{6,10,11}$ A controlled trial by Chadwick et $\mathrm{al}^{5}$ in the UK tested the effectiveness of 5 -session mindfulness groups followed by home practices for 11 schizophrenia sufferers with distressing voices and paranoia on subjective well-being (mindfulness), symptom severity, and life functioning immediately after intervention. There is an increasing interest to find out whether mindfulness-based intervention can be important for people with schizophrenia who are often characterized by unexpectedly low adherence to treatments, or only partially responsive to standard treatment and/or psychosocial interventions. These characteristics lead to a chronic course of illness and frequent relapses. ${ }^{3}$

Findings of two controlled trials also suggest that Acceptance and Commitment Therapy with a strong mindfulness component can significantly reduce psychotic patients' rehospitalization. ${ }^{9}, 12$ These findings provide better understanding of mediating the patients' distress in response to psychotic symptoms. This is opined to be a premise that patients are distressed not by the symptoms but by the meaning they constructed for these symptoms and subsequent emotional responses. ${ }^{9}, 13$

Williams et $\mathrm{al}^{14}$ suggested that the positive effects of mindfulness programs could be mediated/attributed by reductions of overgeneral memory and ruminative thinking and improvements in meta-awareness and specificity of describing psychiatric symptoms, which in turn might lead to much decrease in negative thoughts and depressive or anxiety reactions. A few recent case/cohort and quasi-experimental (nonrandomized) studies of mindfulness-based intervention in schizophrenia reported short- to medium-term patient outcomes (eg, $<6$ months), ${ }^{6,15,16}$ suggesting its potential effectiveness to reduce distressing thoughts or images and disorganization of thoughts.

Nevertheless, most mindfulness interventions have been developed and tested in Western populations; it is uncertain whether this approach to psychosocial intervention can be applicable to Asian/Chinese populations who have an external locus of control and less self-affirmation and openness to experience attitudes. ${ }^{17,18}$ With these knowledge gaps in mind, a mindfulness-based psychoeducation intervention was designed and tested in Hong Kong Chinese people with schizophrenia spectrum disorders to evaluate its effects on a wide variety of patient outcomes such as psychotic symptoms, rehospitalizations, functioning, and insight into the illness.

\section{Aims and hypotheses}

This randomized controlled trial tested the effects of a mindfulness-based psychoeducation group program (MPGP) (in addition to usual psychiatric care) for Chinese patients with schizophrenia spectrum disorders, in comparison to one conventional psychoeducation group program (CPGP) or treatment-as-usual (TAU) only, over a 6-month follow-up. Primary outcomes were the participants' functioning and rehospitalization rate, whereas secondary outcomes included these patients' psychotic symptoms, insight into illness, and progress of recovery. We hypothesized that when compared with those in the CPGP and TAU alone, the MPGP participants could indicate significantly greater improvements over the 6-month follow-up in the following:

1. their functioning and rehospitalization rates (primary outcomes);

2. their insight into illness/treatment, symptom severity, and progress of recovery.

\section{Methods}

The multicenter, controlled trial adopted an assessor-blind, repeated-measures and three-arm design (subject recruitment between June and November 2014 and 6-month follow-up between December 2014 and October 2015). An intentionto-treat (ITT) principle was used for data analysis and all participants were assessed with the same set of outcome measures over the follow-up period, regardless of the levels 
of completion of or adherence to their intervention assigned. ${ }^{19}$ This controlled trial was registered at ClinicalTrials.gov (Ref: NCT01667601; https://register.clinicaltrials.gov). Flow diagram of the trial procedure is presented in Figure 1, according to the latest CONSORT statement. ${ }^{20}$

\section{Participants and settings}

The controlled trial was conducted at two psychiatric outpatient clinics in Hong Kong. About 1,200 patients with schizophrenia spectrum disorders (eg, schizophrenia and schizophreniform disorders) were attending the two

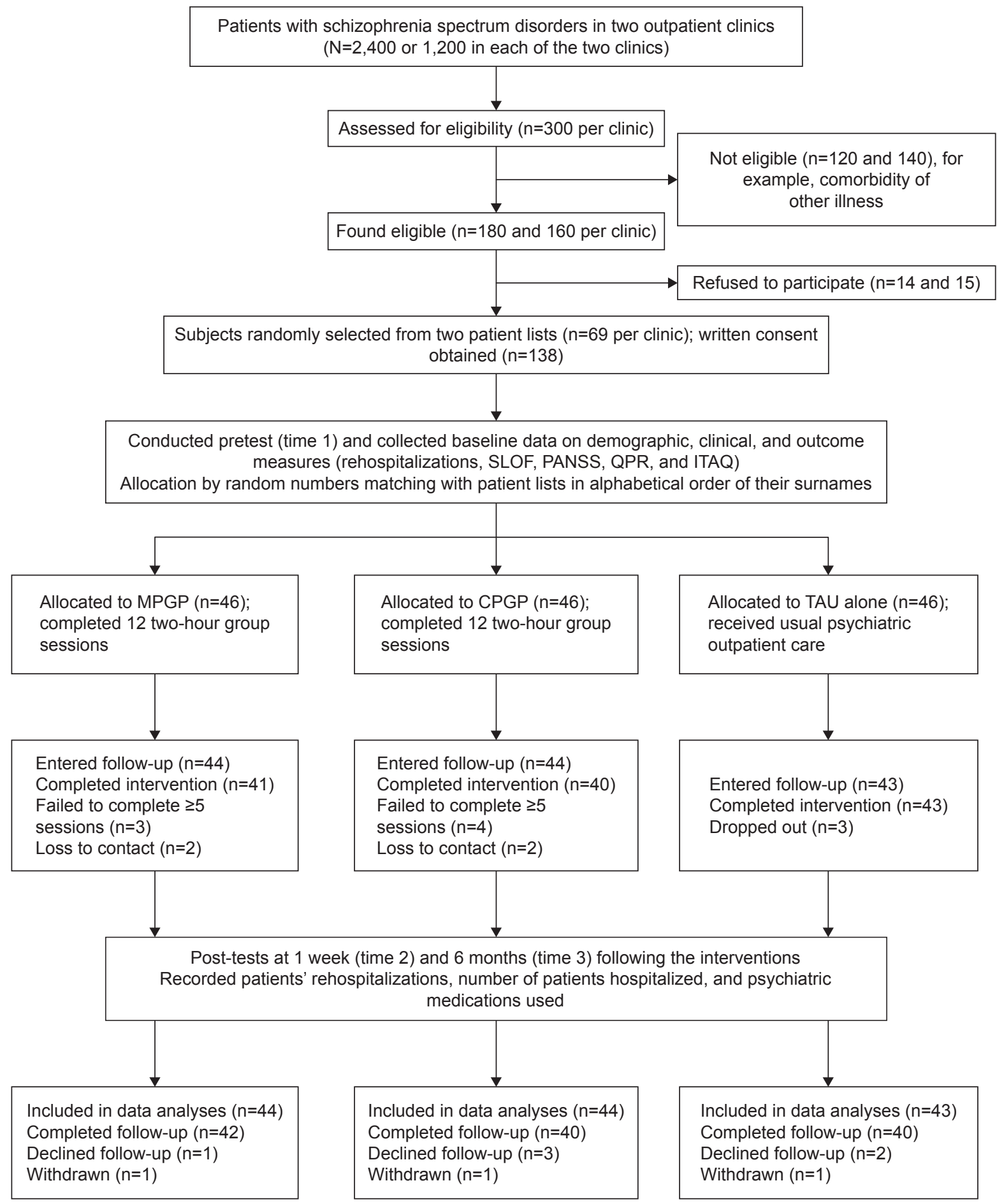

Figure I Flow diagram of the procedure of the controlled trial.

Abbreviations: CPGP, conventional psychoeducation group program; ITAQ, Insight and Treatment Attitudes Questionnaire; MPGP, mindfulness-based psychoeducation group program; PANSS, Positive and Negative Syndrome Scale; QPR, Questionnaire for the Process of Recovery; SLOF, Specific Level of Functioning Scale; TAU, treatment-as-usual. 
outpatient clinics in each of the two clinics under study $(\mathrm{N}=2,400)$ at recruitment. Of $300(25 \%)$ screened for eligibility in each clinic, $180(60 \%)$ and $160(55 \%)$ were found eligible and agreed to participate. As the sample size was planned to be 138 subjects, 69 (38\% and 43\%) were then randomly selected from each of the two clinics. At the clinics, all eligible patients were listed in terms of alphabetical order of their surnames; 69 of them were randomly selected from each patient list according to the computer-generated random numbers provided by an independent statistician, in which 14-15 (18\%) of patients approached in each clinic refused to participate in this trial because of lack of interest and time to participate. With informed written consent obtained, the 144 patients were invited and 138 agreed to participate (ie, response rate $=95.8 \%$ ).

Participants' inclusion criteria included those Chinese outpatients who were 1) aged 18-60 and diagnosed with schizophrenia or its subtypes according to the Diagnostic and Statistical Manual of Mental Disorders, Fourth Edition, Text Revision; ${ }^{21}$ 2) not $>5$ years of schizophrenia at recruitment; and 3) able to understand and communicate in Cantonese/ Mandarin/Chinese languages. Those outpatients were excluded if they got comorbidity of organic brain disorder and learning disability, were found mentally unstable or unfit for study participation by their psychiatrist, and/or had recently received (been receiving) psychoeducation or any structured psychosocial intervention(s).

After completed baseline measurement, the participants were randomly assigned into one of the three arms by using a set of computerized random numbers generated by the statistician with a stochastic minimization program (balanced gender and symptom severity [Positive and Negative Syndrome Scale score $\{$ PANSS $\}$ ] between groups). ${ }^{22}$

\section{Instruments}

The primary outcomes of this study were patient functioning, Specific Level of Functioning Scale (SLOF), ${ }^{23}$ and average number and length of patients' rehospitalizations in the past 6 months.

The 43-item SLOF measured the patients' levels of psychosocial functioning in schizophrenia, ${ }^{23}$ in terms of self-maintenance, social functioning, and community living skills. The scale was scored on a 5-point Likert scale (1, "fully dependent" to 5, "fully self-sufficient"). In schizophrenia, the Chinese version showed very good content validity, internal consistency with Cronbach's alpha of $0.90-0.95$, and testretest reliability (intraclass correlation $=0.84-0.88$ ). ${ }^{24,25}$

The PANSS assessed the symptom severity in psychoses and its 30 items were rated on an 8-point Likert scale
(0, "absent" to 7 , "extreme"). ${ }^{22}$ The scale demonstrated satisfactory concurrent validity with other symptom scales ( $r=0.85-0.90)$, internal consistency with Cronbach's alpha of $0.87-0.92$, and test-retest reliability (intraclass correlation of 0.85-0.90) in Chinese psychotic patients. ${ }^{24}$

The secondary outcomes composed patients' levels of recovery, Questionnaire about the Process of Recovery (QPR), ${ }^{26}$ and insight into illness/treatment, Insight and Treatment Attitudes Questionnaire (ITAQ), ${ }^{27}$ psychotic symptoms, and PANSS. ${ }^{28}$

Patient's level of recovery was measured with the $\mathrm{QPR}^{26}$ with a panel of psychotic patients. The QPR was translated and validated by the research team, ${ }^{17}$ consisting of 22 items and three subscales (self-empowerment, effective interpersonal relationships, and rebuilding life). Its items were rated on a 5-point rating scale ( 0 , "disagree strongly" to 4 , "agree strongly"). The Chinese version demonstrated very satisfactory internal consistency with Cronbach's alphas of $0.88-0.90$, sensitivity to contrasting (symptom severity) groups ( $t=4.68$, $P<0.01$ ), and test-retest reliability at 2 -week interval (intraclass correlation $=0.87-0.92$ ) in psychotic patients. ${ }^{17,29}$

The ITAQ consisted of 22 items and assessed patients' awareness and acceptance of mental illness and prescribed treatments, ${ }^{27}$ scoring on a 3-point Likert scale ( 0 , "no insight" to 2, "good insight"). The Chinese ITAQ indicated very acceptable content validity, inter-rater reliability (intraclass correlation $=0.82$ ), and internal consistency with Cronbach's alpha of $0.82 . .^{30}$

All outcome measures, including those Chinese versions (SLOF, QPR, and ITAQ), were validated and found reliable and valid in Chinese psychotic patients. ${ }^{17,24,25}$ In addition, at the three post-tests, Five Facet Mindfulness Questionnaire (FFMQ) was used to assess the participants' levels of performance or practices made on five components of mindfulness skills (observing, describing, performing with awareness, not giving judgment of inner experiences, and not over-reacting to these experiences). ${ }^{6}$ Its items were rated on a 5-point scale (1, "very rarely true" to 5, "always true"). The FFMQ indicated satisfactory internal consistency (Cronbach's $\alpha=0.76-0.92$ ) and predictive power to psychological well-being and symptoms. ${ }^{31}$

\section{Treatments}

The MPGP consisted of 12 two-hour group sessions (1215 patients per group) every 2 weeks (ie, totally 24 weeks). It was developed from the Kabat-Zinn's MBSR program and modified for community mental health care service in Hong Kong. ${ }^{30}$ The content of a psychoeducation group intervention validated in Hong Kong Chinese psychotic patients by 
Chan et $\mathrm{al}^{32}$ and Chien and Bressington ${ }^{33}$ was also integrated into the MPGP. One trained psychiatric advanced practice nurse (with 5 years of psychiatric rehabilitation and 3 years of mindfulness group experiences) facilitated all the treatment groups in the two clinics. The mindfulness theory used in the MPGP targeted at enhancing patients' awareness, acceptance, and management of their illness and psychotic symptoms; improving their insight into the illness/treatment; resolving the illness-related problems and their poor functioning, helpseeking, and self-care.

The program contained seven domains, including 1) program overview and engagement; 2) enhancing awareness and understanding of bodily sensations, thoughts, and feelings regarding illness experiences or symptoms; 3) guided body awareness and mindful exercises and homework practices; 4) education workshop of illness (schizophrenia) management; 5) encountering with and controlling negative thoughts and perceptions, and life difficulties caused by symptoms, and practicing most effective problem-solving strategies; 6) behavioral rehearsals of means for relapse prevention; and 7) effective mindfulness practices, utilization of community support resources, and making realistic future plans. The MPGP could also promote an individual's restructure of individual thoughts and emotions in specific traditional Chinese culture (beliefs and behaviors). For instance, during the early sessions, participants were encouraged to practice regularly (not less than twice daily and 20 minutes per practice) of focused attention of their bodily sensations, thoughts and feelings, and mindful exercises (on breathing and walking), in which the facilitator also focused on understanding their Chinese cultural beliefs/behaviors - strong interdependence, encouraging better mutual support, practicing expression of feelings openly, and exploring ways of looking at life situations among group members. Participants were also encouraged to cultivate an open/accepting attitude and positive thinking/responses to life problems and thus develop a "decentered" (passing events in mind) attitude on their thoughts/feelings. ${ }^{9,13}$ Details of the MPGP can be provided by the authors upon request.

The CPGP also consisted of 12 two-hour sessions (every 2 weeks). The program adopted the group psychoeducation manual established by Chan et $\mathrm{al}^{32}$ and Lehman et $\mathrm{al}^{34}$ PORT program in the USA. Participants received education and psychological support by one advanced practice nurse (having 6 years of experiences in psychiatric rehabilitation). The nurse was trained by the research team and one rehabilitation specialist (social worker) with a 2-day workshop, consisting of lectures (1 hour each), discussion, sharing experiences, and supervised practices for group sessions. The CPGP consisted of four phases: 1) program overview, engagement, and setting goals for illness management (two sessions); 2) mental health education and information sharing about schizophrenia and survival skills training (four sessions); 3) relapse prevention, resilience promotion, and life skills training (four sessions); and 4) reviews on learned knowledge and skills and establishing future plans (two sessions).

All of the group sessions were tape-recorded (with participants' consent) and reviewed for monitoring the progress and fidelity of the two programs between two sessions, by the research team, and then, they discussed and solved the problems encountered in the group sessions.

TAU comprised the routine psychiatric outpatient care received by all of the 138 participants, which was found similar between the two clinics. The services mainly included psychiatric consultation, treatments, and referrals for medical or specialized care by psychiatrist; brief education and information sharing about the illness and its treatments, and available health care services by nurses; advices/referrals on social welfare and finance by medical social worker; and individual/family counseling by clinical psychologist, whenever necessary.

\section{Data collection procedure}

The Human Research Ethics Committee of The Hong Kong Polytechnic University (HSEARS20140218003) and the two clinics granted the ethical approvals for this controlled trial. Voluntary participation was assured from each patient with informed written consent before recruitment made. All potential participants were asked for any clarifications needed, and their questions were responded with their satisfaction. Confidentiality of the participants' personal identity and data collected were assured and their right for refusal of or withdrawal from the study at all times without negative impact on their treatment plans.

After securing the patients' consents, the research assistant who was blind to the group allocation administered the baseline measurements (time 1) to the individual participants in the clinics and they were then randomly allocated into one of the three study arms by the first author. The participant lists were safely kept in a locked cabinet and concealed to the researchers, clinic staff, and outcome assessor (research assistant) and the research assistant (blind to the group assignment) performed all of the outcome measurements and data entries, in order to avoid subjective bias. ${ }^{35}$ To minimize treatment contamination, the first author asked the participants not to discuss about their program received to clinic staff and copatients. ${ }^{35}$ The last author concealed to sampling/intervention procedures checked the accuracy of all data entries. 
After that, all participants undertook the assigned 6-month intervention, and during the outpatient clinic follow-up, the research assistant conducted the outcome measurements (two post-tests): 1-week (time 2) and 6-month (time 3) postintervention. The frequency and length of psychiatric readmissions, and the total patients (per group) hospitalized in the past 6 months, were examined from the patient records system of the clinics. Types and dosages of psychotropic drugs were also recorded; and facilitating the comparison of the dosages of antipsychotics between groups and/or across studies, the dosages were converted into their haloperidol equivalents. ${ }^{36}$ While no psychoeducation or mindfulness intervention was found within TAU, the participants reported any structured psychological interventions received during the intervention and follow-up period.

The manuals of both the MPGP and CPGP were validated by an expert panel (two psychiatrists, four specialists in psychiatric rehabilitation from allied health professions such as mental health nurse, occupational therapist, and clinical psychologist, and two volunteer peer mentors or ex-patients). The panel assessed the clarity and appropriateness on the topics, case examples, and practices of each manual independently. Most of the topics in these manuals were rated "very satisfactory" or "excellent" on both the clarity (90\%-98\%) and appropriateness (91\%-96\%). Only a few items were reworded on their ways/styles of expression, format, and/or terminology. Intervention fidelity was assessed with a checklist to assess adherence to the topics and instructions, as recommended by the National Institute of Health Behaviour Change Consortium. ${ }^{37}$ The research team rated on the group facilitator's adherence to the manual items by viewing each tape-record against the checklist.

\section{Sample size calculation}

Sample size estimation was made with reference to a few clinical trials of psychoeducation or mindfulness-based programs for people with psychotic disorders. ${ }^{8,10,17,24}$ The effect sizes of patient functioning and rehospitalization rates were between 0.48 and 0.86 in comparison to usual care only over a 6-month follow-up. In addition, one pilot three-arm controlled trial of mindfulness intervention for Chinese people with schizophrenia indicated that the effect sizes of level of functioning and length of readmissions at post-test ( 1 week following intervention) were 0.60 and 0.50 , respectively. ${ }^{30}$ As the result, a total of 138 patients $(n=46$ per group) were required to detect any statistical differences on patient functioning and length/number of rehospitalizations between three study groups at moderate effect sizes of 0.50 , power of 0.80 (two-sided, $P<0.05$ ), as well as a potential attrition rate of $20 \% .^{10,24,30}$

\section{Data analysis}

According to the principles of ITT, SPSS (IBM) for Windows, version 22, was used to insert, check, and analyze the data of patients' demographic and clinical characteristics and outcome variables. Homogeneity of study groups was examined by comparing the participants' characteristics and baseline outcome scores between three groups and settings (two clinics or cities) using one-way analysis of variance (ANOVA) or chi-square tests (for dichotomous data). Any of these variables found significant different between groups would be treated as covariant(s) in the analyses of the treatment effects. However, there were not any significant correlation found on any of the characteristics and baseline data between groups or settings. Recognizing minimal violations of principles of multivariate model of analysis such as multivariate normality and equality of variance-covariance, the interaction (Group $\times$ Time) treatment effects were examined using the mixed-model multivariate or multivariate analyses of variance (MANOVA) test. ${ }^{19}$ With significant results obtained in the MANOVA test, repeated-measures ANOVA test was then used to compare the patient outcomes (SLOF, rehospitalizations, QPR, ITAQ, and PANSS) and dosage of antipsychotic medication between groups across time (measurements). If these between-group comparisons were found significant, contrast tests (such as Helmert's contrasts) were performed to examine any significant mean score difference (MD) (between-group) on individual outcomes (if found statistically significant in ANOVA test). ${ }^{19,38}$ Subgroup differences of the MPGP between two clinics, two cities, and those with low ( $<6$ sessions) and high ( $\geq 6$ sessions) attendance on the above outcomes showing significant results were also found, using ANOVA tests. Total number of patients rehospitalized over 6 months was compared between groups across measurements, using Kruskal-Wallis $H$ test (if found significant, then analyzed by Mann-Whitney $U$ test). The mean total scores of the FFMQ in the MPGP participants were compared between two post-tests. The level of statistical significance was set at 0.05 .

\section{Results}

\section{Characteristics of study participants}

There were 131 of the 138 participants (95\%) who completed the interventions assigned (ie, failed to complete at least 5 sessions) and at least 1 post-test, and thus their data were included in final data analyses. Two in the MPGP 
and CPGP alone and three in the TAU only were unable to contact at 1 week after the interventions (time 2), and two to four participants in the three study groups declined the follow-up (time 3) or withdrew from the study. Only those participants who could not complete both post-tests (times 2 and 3) were not counted in the analysis of treatment effects (Figure 1). Reasons for withdrawal from participation and incompletion of the interventions were similar, including time inconvenience for attending the intervention $(n=4)$, lack of interest to continue their study participation $(n=4)$, and very poor mental condition $(n=2)$. Intervention fidelities for the MPGP and CPGP were very promising, ranging from $88.5 \%$ to $94.3 \%$ (average percentage of agreement $=92.0 \%$ ) and from $89.5 \%$ to $96.0 \%$ (average agreement $=93.5 \%$ ) in adherence to the intervention protocols, respectively.

The participants' sociodemographic and clinical characteristics ( $\mathrm{n}=46$ per study group) are summarized (Table 1 ). Their mean ages were 23.8-25.0 years (standard deviation $[\mathrm{SD}]=6.3-7.0)$, ranging from 18 to 39 years. Over $82 \%$ received oral antipsychotic medications with low/medium dosage (haloperidol equivalents ranged $3.0-8.5 \mathrm{mg} /$ day).$^{36}$ About 50\% were taking second-generation or blended mode (both first- and second-generation) of antipsychotic medications. There were no significant differences found on all of the characteristics between the three study groups (whereas $P=0.18-0.38$ ) as shown in Table 1 , as well as between two clinics under study $(P>0.14)$. In addition, there were no correlations between these characteristics and the baseline outcome scores (Spearman's $r_{\mathrm{p}}<0.11$ ), indicating minimal covariate effect to the outcome variables.

\section{Treatment effects}

Mean (and SD) values of baseline outcome scores and two post-tests for the participants are presented in Table 2. There were no significant differences on the baseline mean scores of all study outcomes between the three groups ( $P=0.20-0.31$ ). There were only eight sets of missing data (mainly on SLOF, QPR, and ITAQ) found on the outcomes of two to four participants in the three groups at time 2 or 3 , and these created very minimal differences in the results of the outcome analyses when tested by different missing data management strategies. Hence, these missing data were filled up by bringing forward individual participants' previous data in the final data analysis. ${ }^{38}$

There were no violations of the assumptions for multivariate analysis of outcome variables (eg, nonsignificant homogeneity of variance-covariance matrices with Box's test at $P=0.18$ and equality of variances with Levene's
Equality of Error Variances test at $P=0.20)$ between groups and low correlations between these outcomes $(r=0.10-0.28)$. Mixed-model MANOVA test was performed, resulting in a significant interactive (Group $\times$ Time) treatment effect on the six outcomes (SLOF, number, and length of rehospitalizations, PANSS, ITAQ, and QPR) between groups $\left(F[6,130]=7.02, P=0.001\right.$, Wilks' $\lambda=0.85$; partial $\left.\eta^{2}=0.54\right)$, with a large effect size. ${ }^{38}$ When between-group effects for individual outcome variables were assessed independently ( $F$ values in Table 2$)$, the MPGP showed significant greater improvements on four study outcomes, including patients' functioning $(F[2,129]=6.98, P=0.0009)$ and its subscales $(P=0.003-0.0007)$; psychotic symptom severity $(F[2,129]=6.20, P=0.003)$ and both positive and negative symptom subscales ( $P=0.001$ and 0.005 , respectively), progress on recovery $(F[2,129]=5.83, P=0.005)$, and insight into their illness/treatment $(F[2,129]=5.10, P=0.01)$, than the CPGP and/or TAU alone.

The MDs of the above four outcomes in contrast tests indicated significant differences between groups as follows:

- Level of functioning (SLOF) in the MPGP significantly better improved at times $2-3(\mathrm{MD}=16.8$ and 30.6, $P=0.008$ and 0.0009 , respectively) in comparison to TAU alone, whereas the MPGP also indicated greater improvements in their functioning at the post-tests than the CPGP $(P=0.02$ and 0.006). In addition, the CPGP indicated greater functional improvements at times 3 and 4 (MD =7.9 and 11.4, $P=0.05$ and 0.01 ) than the TAU alone.

- Psychotic symptoms (PANSS score) in the MPGP significantly reduced at time $2-3(\mathrm{MD}=8.5$ and $18.5, P=0.01$ and 0.003 , respectively), whereas the TAU alone indicated consistent increase in symptom severity across time (from $\mathrm{M}=88.1 \pm 9.0$ at baseline, $\mathrm{M}=90.2 \pm 10.1$ at time 1 to $\mathrm{M}=97.8 \pm 10.8$ at time 2 ). The MPGP also indicated significant greater reduction of psychotic symptoms than the CPGP at time $3(\mathrm{M}=70.0 \pm 10.0$ vs $\mathrm{M}=84.1 \pm 9.5)$.

- Progress of recovery from the illness (QPR score) in the MPGP participants significantly better improved at times 2 and $3(\mathrm{MD}=3.4$ and $8.3, P=0.01$ and 0.0008 , respectively) than the TAU-alone group, whereas the CPGP participants indicated significantly better recovery condition than the TAU alone group at time 3 $(\mathrm{MD}=2.5, P=0.04)$. In addition, the MPGP showed significantly better recovery condition than the CPGP at time $3(P=0.001)$.

- Insight into illness/treatment (ITAQ score) of the MPGP significantly greater improved at time $3(\mathrm{MD}=5.4$, $P=0.001)$ than both the CPGP and TAU alone, whereas 
Table I Characteristics of MPGP, CPGP, and TAU participants $(\mathrm{N}=138)$

\begin{tabular}{|c|c|c|c|c|c|}
\hline Characteristics & $\begin{array}{l}\text { MPGP }(n=46), f(\%) \\
\text { or M } \pm \text { SD }\end{array}$ & $\begin{array}{l}\text { CPGP }(n=46), f(\%) \\
\text { or } M \pm S D\end{array}$ & $\begin{array}{l}\text { TAU }(n=46), f(\%) \\
\text { or } M \pm \text { SD }\end{array}$ & $\begin{array}{l}\text { Chi-square or } \\
\text { ANOVA test }{ }^{\mathrm{a}}\end{array}$ & $P$-value \\
\hline Gender & & & & $\chi^{2}=1.04$ & 0.38 \\
\hline Male & $24(52.1)$ & $23(50.0)$ & $25(54.3)$ & & \\
\hline Female & $22(47.8)$ & $23(50.0)$ & $21(45.7)$ & & \\
\hline Age (years) & $\begin{array}{l}23.8 \pm 6.8 \\
\mathrm{Cl}=16.9-30.3\end{array}$ & $\begin{array}{l}24.1 \pm 6.3 \\
C l=17.6-30.6\end{array}$ & $\begin{array}{l}25.0 \pm 7.0 \\
C l=\mid 7.8-31.9\end{array}$ & $F=1.13$ & 0.30 \\
\hline $18-25$ & $18(39.1)$ & $15(32.6)$ & $18(39.1)$ & & \\
\hline $26-30$ & $15(32.6)$ & $18(29.1)$ & $16(34.8)$ & & \\
\hline $31-35$ & $10(21.7)$ & $9(19.6)$ & $9(19.6)$ & & \\
\hline$>35$ & $3(6.5)$ & $4(8.7)$ & $3(6.5)$ & & \\
\hline Education level & & & & $\chi^{2}=1.31$ & 0.33 \\
\hline Primary school or below & $8(17.4)$ & $7(15.2)$ & $7(15.2)$ & & \\
\hline Secondary school & $30(65.2)$ & $30(65.2)$ & $29(63.0)$ & & \\
\hline University or above & $8(17.4)$ & $9(19.6)$ & $10(21.8)$ & & \\
\hline Primary diagnosis & & & & $\chi^{2}=1.25$ & 0.28 \\
\hline Schizophrenia & $20(43.5)$ & $19(4 I .3)$ & $19(4 \mid .3)$ & & \\
\hline Schizophreniform disorder & $12(26.1)$ & $13(28.3)$ & $12(26.1)$ & & \\
\hline Schizoaffective disorder & $10(21.7)$ & $10(21.7)$ & $9(19.6)$ & & \\
\hline Other psychotic disorders & $4(8.7)$ & $4(8.7)$ & $6(13.0)$ & & \\
\hline Monthly household income (HKD) ${ }^{b}$ & $\begin{array}{l}|5,08| \pm 4,0 \mid 3 \\
C l=||, 523-19,205\end{array}$ & $\begin{array}{l}|6,7| 2 \pm 4,702 \\
C l=||, 60 \mid-20,520\end{array}$ & $\begin{array}{l}|7,005 \pm 3,98| \\
\mathrm{Cl}=\mid 3,257-20,995\end{array}$ & $F=I .40$ & 0.25 \\
\hline 10,000 or below & $7(15.2)$ & $7(15.2)$ & $6(13.0)$ & & \\
\hline$|0,00|-20,000$ & $17(37.0)$ & $16(34.8)$ & $16(34.8)$ & & \\
\hline $20,00 I-30,000$ & $18(39.1)$ & $20(43.5)$ & $20(43.5)$ & & \\
\hline$>30,000$ & $4(8.7)$ & $3(6.5)$ & $4(8.7)$ & & \\
\hline Duration of illness (years) & $\begin{array}{l}2.0 \pm 1.0, \text { range: } 0.2-3.0 \\
\mathrm{Cl}=0.9-3.0\end{array}$ & $\begin{array}{l}\text { 2. } I \pm 0.9 \text {, range: } 0.4-3.0, \\
\mathrm{Cl}=1.2-3.1\end{array}$ & $\begin{array}{l}2.0 \pm 0.9, \text { range: } 0.5-2.9 \\
C l=I . I-3.0\end{array}$ & $F=1.45$ & 0.24 \\
\hline$<1$ & $14(30.4)$ & $13(28.3)$ & $13(28.3)$ & & \\
\hline $1-2$ & $14(30.4)$ & $15(32.6)$ & $15(32.6)$ & & \\
\hline $2-3$ & $10(21.8)$ & $10(21.8)$ & II (23.9) & & \\
\hline $3-5$ & $8(17.4)$ & $8(17.4)$ & $7(15.2)$ & & \\
\hline $\begin{array}{l}\text { Number of family members living with } \\
\text { patient }\end{array}$ & & & & $\chi^{2}=1.65$ & 0.23 \\
\hline None & $10(21.7)$ & $9(19.6)$ & $9(19.6)$ & & \\
\hline $1-2$ & $29(63.1)$ & $28(60.9)$ & $30(65.2)$ & & \\
\hline $3-4$ & $7(15.2)$ & $9(19.6)$ & $7(15.2)$ & & \\
\hline Type of medication & & & & $\chi^{2}=1.23$ & 0.28 \\
\hline Conventional antipsychotics (eg, haloperidol) & $16(34.8)$ & $15(32.6)$ & $17(37.0)$ & & \\
\hline Atypical antipsychotics (eg, risperidone) & $18(39.1)$ & $18(39.1)$ & $17(37.0)$ & & \\
\hline Antidepressants (eg, fluoxetine) & $7(15.2)$ & $6(13.1)$ & $7(15.2)$ & & \\
\hline Blended mode ${ }^{c}$ & $5(10.9)$ & $7(15.2)$ & $5(10.9)$ & & \\
\hline Use of psychiatric services & & & & $\chi^{2}=1.82$ & 0.20 \\
\hline Medical consultation and treatment planning & $43(93.5)$ & $46(100.0)$ & $44(95.7)$ & & \\
\hline Nursing advice on services and brief education & $40(87.0)$ & $39(84.8)$ & $40(87.0)$ & & \\
\hline Social welfare and financial advices & $38(82.6)$ & $37(80.4)$ & $36(78.3)$ & & \\
\hline Individual/family counseling & $20(43.5)$ & $18(39.1)$ & $15(32.6)$ & & \\
\hline Dosage of medication ${ }^{d}$ & & & & $\chi^{2}=1.98$ & 0.18 \\
\hline High & $10(21.7)$ & $10(21.7)$ & II (23.9) & & \\
\hline Medium & $20(43.5)$ & $19(41.3)$ & $20(43.5)$ & & \\
\hline Low & $16(34.8)$ & $17(37.0)$ & $15(32.6)$ & & \\
\hline
\end{tabular}

Notes: aAOVA test ( $F$-test, $d f=136$ ) was used to compare the participants' sociodemographic variables in interval or continuous level of measurement between three study groups; otherwise, chi-square tests were used. bUS\$I=HKD 7.80. 'Patients were taking more than one type of psychotropic medication such as the use of either conventional and atypical antipsychotic or atypical antipsychotic together with one antidepressant. ${ }^{\mathrm{D}}$ Dosage levels of psychotropic/antipsychotic medications were compared with the average dosage of medication taken by the patients in haloperidol-equivalent mean values. ${ }^{36}$

Abbreviations: ANOVA, analysis of variance; $\mathrm{Cl}, 95 \%$ confidence interval; CPGP, conventional psychoeducation group program; f, frequency; M, mean; MPGP, mindfulnessbased psychoeducation group program; SD, standard deviation; TAU, treatment-as-usual or usual psychiatric outpatient care. 


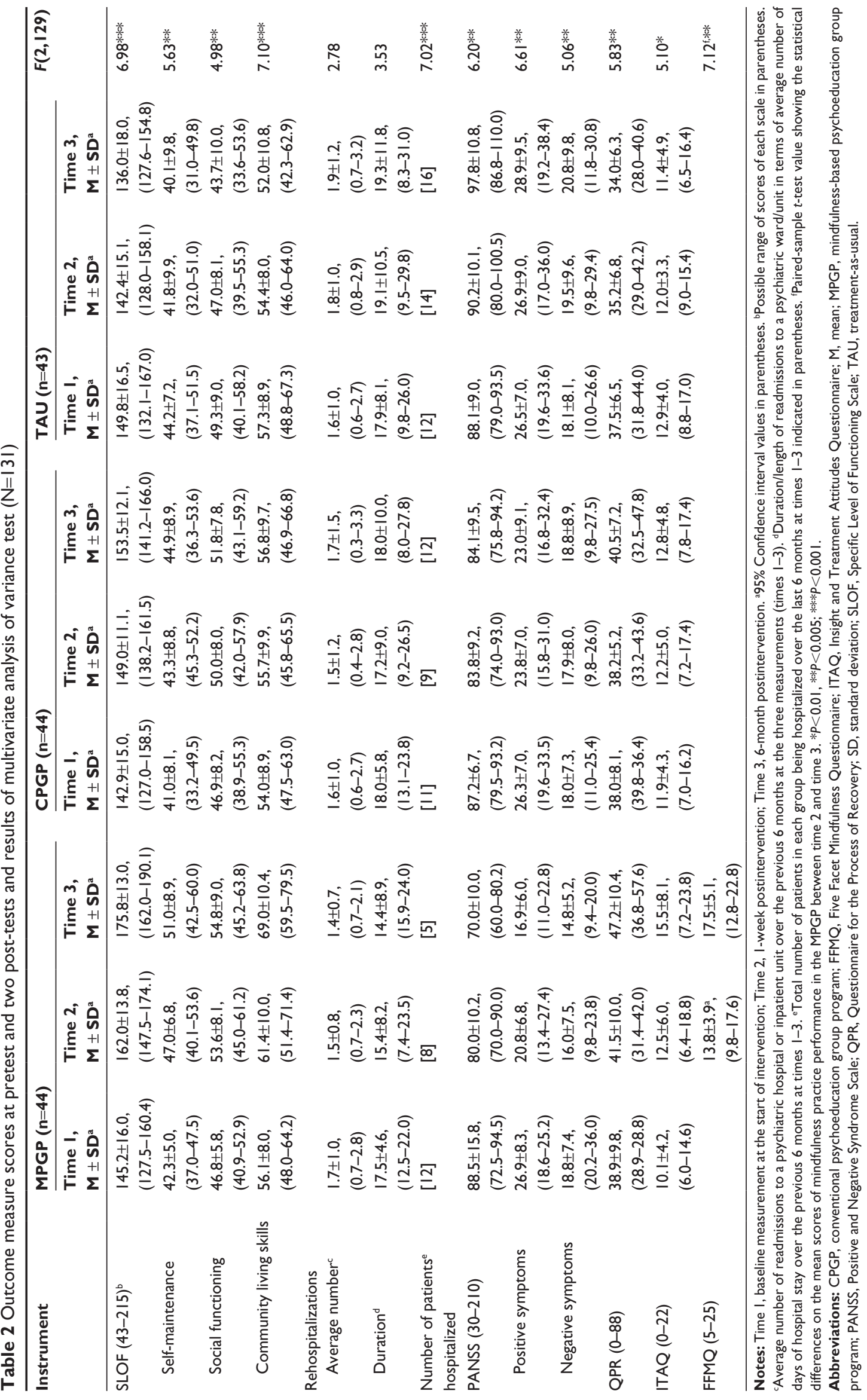


the CPGP and TAU alone group indicated very mild changes in their insight scores.

Furthermore, the MPGP had significantly less patients being rehospitalized into psychiatric units over the past 6 months than both the CPGP and TAU alone at time 3 (Kruskal-Wallis test value $=8.41, P=0.005,5[11 \%]$ vs 12 [27\%] for CPGP vs 16 [37\%] for TAU alone). The significant outcomes mentioned above (SLOF, PANSS, QPR, and ITAQ) did not show any significant differences between the two clinics $(P=0.15-0.26)$ and between the MPGP with low ( $<6$ sessions) and high ( $\geq 6$ sessions) attendance $(P=0.10-0.20)$, using repeated-measures ANOVA tests. As shown in Table 2, the mindfulness performance (FFMQ score) in the MPGP significantly improved from time 1 to time 2 (paired $t=7.12, P=0.003$ ). However, there were no significant differences on the dosages and types of antipsychotic medications and psychosocial interventions used (eg, family/individual psychotherapy and medication management) between groups over follow-up $(P>0.25)$.

\section{Discussion}

This randomized controlled trial was one of the few conducted to test the effects of a MPGP for people with schizophrenia spectrum disorders and its comparative effectiveness with a common and well-accepted psychoeducation group program. The MPGP for these Chinese patients demonstrated more superior and wider variety of positive results than the CPGP, as well as the usual psychiatric care alone. It is highly encouraging and important to find out that the MPGP can produce significantly greater improvements in different aspects of patient functioning (including self-maintenance, social interactions, and living skills), both positive and negative (psychotic) symptoms, and insight into their illness and treatments over the 6-month follow-up, with moderate large effect sizes. The MPGP can also enhance these patients' progress of recovery (eg, accepting responsibilities and tasks to rebuild one's life and ability to engage in self-reflection on the ways that recovery is influenced by one's interpersonal relationships). ${ }^{26}$

Despite that the MPGP could not demonstrate its effect on reducing readmissions significantly, the average number and duration of rehospitalizations among the MPGP participants progressively decreased over the follow-up period, whereas the other two study groups had slightly changes or increases in these rehospitalization rates across time. In consonance with these positive results, the MPGP participants could continuously and significantly improve their mindfulness performance (FFMQ scores) from postintervention to the 6-month follow-up. These findings may fill in the knowledge gap suggested by recent systematic reviews that the CPGPs might show significant short- to medium-term effects in mainly symptom control or improving mental state and relapse prevention in schizophrenia and its spectrum disorders. . $^{2,89}$

It is important to note that the MPGP in this study can also exert moderately large effects on negative symptoms, which has rarely been found in most of the current psychiatric treatments or psychosocial interventions in schizophrenia. ${ }^{40}$ As recommended by Coelho et al, ${ }^{11}$ the therapeutic components of mindfulness (or meditation), including selfawareness, self-empowering, acceptance, and compassion, are integrated into the mindfulness-based intervention to enhance one's motivation to self-regulate or manage their illness, symptoms, and related behaviors, thus being likely to mediate the other patient outcomes. However, the therapeutic mechanisms of mindfulness training to improve the control of psychotic symptoms in schizophrenia are still unknown, thus needing to be explored in future research.

Importantly, the findings can support the benefits of the MPGP in improving patients' psychosocial functioning and inducing their insight into the illness (schizophrenia). In line with the goal of psychiatric treatment and rehabilitation, it is very crucial for psychotic patients to be integrated successfully into their community and living independently there. ${ }^{5,41}$ In addition, psychotic patients can have better adherence to their treatment regimen and more able to perform self-care and management of the illness if their insight into the illness has been enhanced. ${ }^{42}$ Indeed, most of the current psychoeducation or psychosocial interventions have not yet been insight-oriented and thus unable to have significant impact on inducing patients' insight into their illness similar to this MPGP. These findings provide support for combined effects of mindfulness training and psychoeducation in facilitating patients with schizophrenia to be recovered (ie, having symptom alleviation and improvement of functioning, thus achieving fullest capacity and independence of living) at the earliest possible in the community-based rehabilitation. ${ }^{10}$

Previous controlled trials that indicated significant effects $^{5,43,44}$ were mainly designed with quasi-experimental and feasibility or pilot testing, using small-sized samples with more chronic or longer duration of illness and/or comorbidities of other mental illnesses. Those trials were conducted in a single study site and adopted combined approaches to cognitive therapy (eg, meta-cognitive therapy, attention training technique, cognitive-behavioral or acceptance and commitment therapy); they reported higher attritions 
(25\%-50\%) and noncompletion rates $(30 \%-50 \%)$ and used a few narrowly focused patient outcomes (mainly symptom severity and level of mindfulness). This study can provide higher quality of evidence about the effects of mindfulnessbased intervention by using randomized controlled trial design with adequate randomized sample (study power) in early stage of schizophrenia spectrum disorders, low attrition and noncompletion rates, and a wider variety of patient outcomes, when compared with a CPGP. Nevertheless, further multisite, randomized, controlled trials in more diverse patient populations with different comorbidities and a longerterm follow-up, with different structures (eg, individual versus group basis) of the intervention, and/or adopting other clinical outcomes (eg, service utilization and quality of life) are recommended.

In contrast to the common beliefs or cautions about the possible impacts of mindfulness training on exacerbation of psychotic symptoms during meditation practices or mindful exercises, ${ }^{5,13,40}$ this clinical trial provides supporting evidence for the MPGP that can largely improve psychotic patients' mental and psychosocial health conditions and gain insight into the illness, thus reducing risk of their relapse. In this study, there was not any adverse event or reaction relating to the mindfulness-based intervention noted. A few core elements of mindfulness training and psychoeducation might constitute the therapeutic components of the MPGP, including "being aware and accepting/controlling thoughts, feelings and emotions", "coping and problem-solving ability", "self-empowerment and regulation", and "seeking help and support", $4,8,10$ which have not been examined or tested in this study. Therefore, further research is recommended to examine these potential therapeutic components individually or interactively and mechanism of actions of this psychosocial intervention (MPGP) used in this study.

The benefits of the MPGP to these psychotic patients can be started from responding to their symptoms in an intentional and acceptable manner through an improvement of their awareness, acceptance, and relationships with unwanted thoughts and emotions/feelings. Throughout the mindfulness practices, the intervention may help an individual develop cognitive changes (eg, higher acceptance and motivation in rehabilitation and management of the illness), gradually become more focused on the present, and finally relieved from the symptom-related distress. ${ }^{45}$ Recently, there have also been suggestions that mindfulness-based interventions may function through top-down and/or bottom-up mechanisms of cognitive remediation. ${ }^{46}$ Top-down mechanism occurs in the development of interoceptive attention to visceral bodily sensations, facilitating through daily mindful practices such as breathing monitoring. As the meditation practice deepens, interoceptive attention networks increase and alter information processing in the brain to enhance perceptual experience and, at the same time, promote reduced conceptual cortical activity ${ }^{47}$ Bottom-up regulations have also been suggested as modulation of emotion-generative brain regions (limbic) without involving the frontal regions for cognitive regulation (eg, appraisal and suppression). ${ }^{46,48}$ Therefore, it is important to further examine in future research whether the patients who have undertaken the MPGP can experience such cognitive changes and reduce the distress relating to psychotic symptoms; hence, the results may inform the physiological benefits or a new cognitive remediation model for psychotic symptoms (eg, hallucinations and delusions), as suggested by Freeman et al. ${ }^{49}$

In addition, the MPGP participants in this study could demonstrate significantly higher improvements in most of the primary and secondary outcomes than the CPGP and TAU alone, over 6-month postintervention. Langer et al's ${ }^{43}$ feasibility study of a mindfulness-based cognitive therapy for people with early psychosis also reported significant effects on their ability to respond mindfully to stressful internal events (eg, distressing thoughts and images) at the end of the intervention. However, the patients' symptom severity and unwillingness to come into or stay in contact with internal experience were not significantly improved. Its limitations on adherence to mindfulness training and practices, small sample size, and taking adequate care of patients' educational and resources needs were addressed in this study. Indeed, the MPGP can demonstrate more substantial and diverse therapeutic effects to those patients with early stage of schizophrenia (ie, within 5 years of the illness), in consistent with the findings of Moritz et al' $\mathrm{s}^{44}$ mindfulness intervention trial.

\section{Achieving better participation and completion rates}

While the anticipated benefits were induced by the mindfulness-based practices with the 12 biweekly face-to-face training/education, those review and learning for self-practice sessions may motivate the participants to continue attending and complete the program. However, there can also be a few reasons to possibly explain about such low dropout and noncompletion rates. First, the patients in the early stage of the illness who agreed to participate in the interventions (and study) could be highly motivated to improve their illness condition and much involved in their community rehabilitation or 
any interventions potentially beneficial to the illness. Second, the conditions such as patients being young, highly educated, and having family support and a satisfactory financial situation, together with a culture of respect and trust for professionals such as clinical managers and therapists, ${ }^{33}$ prevailed among Chinese/Asian populations. Last, there might be very few structured psychosocial or mindfulness-based education programs/services available for, or accessible to, Chinese patients with early schizophrenia or psychosis..$^{2,17,33}$

\section{Limitations and implications}

Although this randomized controlled trial has strong internal validity using a random sample from two outpatient clinics in Hong Kong and very satisfactory treatment fidelity, a few main limitations should be noted. First, the participants who had mainly a short duration of illness and were volunteers to participate were very likely to adhere to and complete the intervention and be motivated to get improved in their health outcomes. In addition, all of them were not blind to the intervention allocation, which could produce an expectation or desirable response bias.

Second, the sample consisted of patients with a higher education level, low- to moderate levels of antipsychotic medication, and a better family support. Despite having randomized samples from two clinics, the characteristics of the participants were selective and thus might not be representative of the wider patient (schizophrenia) populations in Hong Kong. To increase the generalization of the findings to these patient populations, the sample could have included those with different chronicity and severity of schizophrenia, as well as those with different comorbidities of other mental disorders. ${ }^{2}$ As mentioned in the above discussion, this selective sample might also contribute to higher levels of completion and lower dropout rates. Therefore, the findings of this study may not be fully generalizable to the Hong Kong/Chinese populations of schizophrenia spectrum disorders, and further research in this patient group with different sociodemographic, illness, and ethnic characteristics is, therefore, recommended.

Third, although the levels of performance in mindfulness practice (FFMQ score) in the MPGP participants have been monitored (ie, significantly enhanced from the postintervention to 6-month follow-up), their adherence and regularity of daily practices could be difficult to standardize/address as what may be achieved by applying a manual- or protocol-driven psychoeducation program. ${ }^{13}$ The extent of engagement and review on mindfulness practices with the therapists might contribute to much psychological and/or treatment effects in these patients; ${ }^{29}$ but these impacts have not been examined. In addition, the individual/combined benefits of multiple components of mindfulness and psychoeducation approaches in the MPGP are not known in this trial. To better understand the therapeutic effects of mindfulness-based intervention and its components, the relationships between patient outcomes and action mechanisms of the MPGP, as well as the potential moderators (eg, symptom severity and duration of and insight into illness) and mediators (eg, acceptance, psychological flexibility, and compassion) of outcomes, ${ }^{11}$ should also be investigated in future controlled trials.

Third, this controlled trial was planned by the research team and conducted by the extensively trained advanced practice psychiatric nurses, thus increasing risk of allegiance bias, ${ }^{50}$ although the study outcomes were measured by an independent assessor. This might also reduce the applicability of the intervention to the usual psychiatric outpatient care that is likely to favor brief and user-friendly or even mobile/ online modes of interventions with simple training. Together with no concealment of intervention participation to clinic staff, the participants could have shared and discussed the study participation/intervention received with the staff and thus might inflate/overstate the treatment effects. ${ }^{20}$

Last, there were changes in the symptom severity but not the remission rates among the participants over 6 months of follow-up. The MPGP participants demonstrating statistically much reduced symptoms over the follow-up than the other study groups might not represent the full symptom remissions (ie, 4-month ratings of all PANSS items as score $\leq 3$ ). ${ }^{51}$ Full remissions among the patients can better reveal their clinically significant and meaningful changes in psychiatric symptoms and thus illness condition or recovery.

\section{Conclusion}

Notwithstanding its limitations, the randomized controlled trial provides important evidence to support the medium-term (6 months) effect of a MPGP for patients with schizophrenia spectrum disorders. Compared with CPGP and usual outpatient care alone, the MPGP participants reported significant positive changes in a few psychosocial outcomes, including improved different aspects of functioning, reduced psychotic symptoms, and enhanced progress of recovery and insight into their illness across the follow-up. This controlled trial supports the use of the MPGP to aid for relapse prevention and improving psychosocial health and functioning, and thus the community-based rehabilitation for patients with early schizophrenia and related disorders. The findings support further research on the mindfulness-based program in 
psychotic disorders with those from wider sociodemographic and clinical characteristics in Asian/Chinese populations, or those with chronic or comorbid mental illness and/or study settings.

\section{Acknowledgments}

The authors would like to thank the Health and Medical Research Fund, Food and Health Bureau (Ref No: 12131641) and the General Research Fund (PolyU 156038/15H), University Grant Council, The Hong Kong SAR's Government for supporting this research project. In addition, we would like to express our gratitude to the patients whose participation made the controlled trial possible and clinic staff for assisting our sample recruitment.

\section{Disclosure}

The authors report no conflicts of interest in this work.

\section{References}

1. Bellack AS. Scientific and consumer models of recovery in schizophrenia: concordance, contrasts, and implications. Schizophr Bull. 2006 32(3):432-442.

2. Chien WT, Yip AL. Current approaches to treatments for schizophrenia spectrum disorders, part I: an overview and medical treatments. Neuropsychiatr Dis Treat. 2013;9:1311-1332.

3. Owen MJ, Sawa A, Mortensen PB. Schizophrenia. Lancet. 2016; 388(10039):86-97.

4. Bäuml J, Froböse T, Kraemer S, Rentrop M, Pitschel-Walz G. Psychoeducation: a basic psychotherapeutic intervention for patients with schizophrenia and their families. Schizophr Bull. 2006;32(Suppl 1): S1-S9.

5. Chadwick P, Hughes S, Russell D, Russell I, Daqnan D. Mindfulness groups for distressing voices and paranoia: a replication and randomized feasibility trial. Behav Cogn Psychother. 2009;37(4):403-412.

6. Baer R. Mindfulness training as a clinical intervention: a conceptual and empirical review. Clin Psychol Sci Practice. 2003;10(2):125-143.

7. Lee S, Chiu MYL, Tsang A, Chui H, Kleinman A. Stigmatizing experience and structural discrimination associated with the treatment of schizophrenia in Hong Kong. Soc Sci Med. 2006;62(7):1685-1696.

8. Xia J, Merinder LB, Belqamwar MR. Psychoeducation for schizophrenia. Cochrane Database Syst Rev. 2011;(6):CD002831.

9. Ma SH, Teasdale JD. Mindfulness-based cognitive therapy for depression: replication and exploration of differential relapse prevention effects. J Consult Clin Psychol. 2004;72(1):31-40.

10. Chlesa A, Serretti A. Mindfulness-based cognitive therapy for psychiatric disorders: a systematic review and meta-analysis. Psychiatry Res. 2011;187(3):441-453.

11. Coelho HF, Canter PH, Ernst E. Mindfulness-based cognitive therapy: evaluating current evidence and informing future research. $J$ Consult Clin Psychol. 2007;75(6):1000-1005.

12. Pfammatter M, Junghan UM, Brenner HD. Efficacy of psychological therapy in schizophrenia: conclusions from meta-analyses. Schizophr Bull. 2006;32(Suppl 1):64-80.

13. Chien WT, Thompson DR. Effects of a mindfulness-based psychoeducation programme for Chinese patients with schizophrenia: two-year follow-up. Br J Psychiatry. 2014;205(1):52-59.

14. Williams JM, Teasdale JD, Segal ZV, Soulsby J. Mindfulness-based cognitive therapy reduces overgeneral autobiographical memory in formerly depressed patients. J Abnorm Psychol. 2000;109(1):150-155.
15. Abba N, Chadwick P, Stevenson C. Responding mindfully to distressing psychosis: a grounded theory analysis. Psychother Res. 2008; 18(1):77-87.

16. Dannahy L, Hayward M, Strauss C, Turton W, Harding E, Chadwick P. Group person-based cognitive therapy for distressing voices: pilot data from nine groups. J Behav Ther Exp Psychiatry. 2011;42(1):111-116.

17. Chien WT, Chan SW. The effectiveness of mutual support group intervention for Chinese families of people with schizophrenia: a randomised controlled trial with 24-month follow-up. Int J Nurs Stud. 2013;50(10):1326-1340.

18. Krushe A, Cyhlarova E, Williams JM. Mindfulness online: an evaluation of the feasibility of a web-based mindfulness course for stress, anxiety and depression. BMJ Open. 2013;3(11):e003498.

19. Stevens JP. Applied Multivariate Statistics for the Social Sciences. $4 \mathrm{ed}$ NJ, USA: Lawrence Erlbaum Associates; 2002.

20. Schulz KF, Altman DG, Moher D; for the CONSORT Group. CONSORT 2010 Statement: updated guidelines for reporting parallel group randomised trials. $B M J .2010 ; 340: \mathrm{c} 332$.

21. American Psychiatric Association. Diagnostic and Statistical Manual of Mental Disorders, The 4th Text Revised Edition (DSM IV-TR). Washington, DC: The APA; 2000.

22. Bell M, Milstein R, Beam-Goulet J, Lysaker P, Cicchetti D. The positive and negative syndrome scale and the brief psychiatric rating scale. Reliability, comparability, and predictive validity. J Nerv Ment Dis. 1992;180(11):723-728.

23. Schneider LC, Struening EL. SLOF: a behavioral rating scale for assessing the mentally ill. Soc Work Res Abstr. 1983;19(3):9-21.

24. Chien WT, Leung SF, Chu CS. A nurse-led, needs-based psychoeducation intervention for Chinese patients with first-onset mental illness. Contemp Nurse. 2012;40(2):194-209.

25. Chien WT, Chan SW, Thompson DR. Effects of a mutual support group for families of Chinese people with schizophrenia: 18-month follow-up. Br J Psychiatry. 2006;189:41-49.

26. Neil ST, Kilbride M, Pitt L, et al. The questionnaire about the process of recovery (QPR): a measurement tool developed in collaboration with service users. Psychosis. 2009;1(2):145-155.

27. McEvoy JP, Apperson LJ, Appelbaum PS, et al. Insight in schizophrenia: its relationship to acute psychopathology. J Nerv Ment Dis. 1989;177(1):43-47.

28. Kay SR, Fiszbein A, Opler LA. The positive and negative syndrome scale (PANSS) for schizophrenia. Schizophr Bull. 1987;13(2):261-276.

29. Chien WT, Chan ZC. Chinese translation and validation of the Questionnaire on the Process of Recovery in schizophrenia and other psychotic disorders. Res Nur Health. 2013;36(4):400-411.

30. Chien WT, Lee IY. The mindfulness-based psychoeducation program for Chinese patients with schizophrenia. Psychiatr Serv. 2013;64(4): 376-379.

31. Baer RA, Smith GT, Hopkins J, Krietemeyer J, Toney L. Using selfreport assessment methods to explore facets of mindfulness. Assessment. 2006;13(1):27-45.

32. Chan SW, Yip B, Tso S, Cheng BS, Tam W. Evaluation of a psychoeducation program for Chinese patients with schizophrenia and their family caregivers. Patient Educ Couns. 2009;75(1):67-76.

33. Chien WT, Bressington D. A randomized controlled trial of a nurse-led structured psychosocial intervention program for people with firstonset mental illness in psychiatric outpatient clinics. Psychiatry Res. 2015;229(1-2):277-286.

34. Lehman AF, Buchanan RW, Dickerson FB, et al. Evidence-based treatment of schizophrenia. Psychiatr Clin North Am. 2003;26(4): 939-954.

35. National Health \& Medical Research Council (NHMRC) Clinical Trials Centre. Randomisation. The CTC Outreach Programme. Australia: University of Sydney; 2013.Available from: http://www.ctc.usyd.edu. au/our-research/ctc-outreach.aspx. Accessed March 1, 2014.

36. Bezchlibnyk-Butler KZ, Jeffries JJ, Procyshyn RM, Virani AS. Clinical Handbook of Psychotropic Drugs. 20th ed. Boston, MA: Hogrefe and Huber; 2013. 
37. Bellg AJ, Borrelli B, Resnick B, et al; Treatment Fidelity Workgroup of the NIH Behavior Change Consortium. Enhancing treatment fidelity in health behavior change studies: best practices and recommendations from the NIH behavior change consortium. Health Psychol. 2004; 23(5):443-451.

38. Tabachnick BG, Fidell LS. Using Multivariate Statistics. 4th ed. New York, NY: Allyn and Bacon; 2001.

39. Xia J, Zhao S, Jayaram MB. Psychoeducation (brief) for people with serious mental illness. Cochrane Database Syst Rev. 2013;(11): CD010823.

40. Khoury B, Lecomte T, Gaudiano BA, Paquin K. Mindfulness interventions for psychosis: a meta-analysis. Schizophr Res. 2013;150(1): 176-184.

41. National Collaborating Centre for Mental Health. Core Intervention in the Treatment and Management of Schizophrenia in Primary and Secondary Care (Clinical Guidelines, No 82). Leicester, UK: British Psychological Society; 2009.

42. Lysaker PH, Buck KD. Insight and schizophrenia: correlates, etiology and treatment. Clin Schizophr Related Psychoses. 2008;2(2): $147-154$.

43. Langer ÁI, Cangas AJ, Salcedo E, Fuentes B. Applying mindfulness therapy in a group of psychotic individuals: a controlled study. Behav Cogn Psychother. 2012;40(1):105-109.

44. Moritz S, Cludius B, Hottenrott B, et al. Mindfulness and relaxation treatment reduce depressive symptoms in individuals with psychosis. Eur Psychiatry. 2015;30(6):709-714.
45. Brown LF, Davis LW, LaRocco VA, Strasburger A. Participant perspectives on mindfulness meditation training for anxiety in schizophrenia. Am J Psychiatric Rehabil. 2010;13(3):224-242.

46. Chiesa A, Seretti A, Jokobsen JC. Mindfulness: top-down or bottom-up emotion regulation strategy? Clin Psychol Rev. 2013;33(1):82-96.

47. Farb NAS, Segal ZV, Anderson AK. Mindfulness meditation training alters cortical representations of interoceptive attention. Soc Cogn Affect Neurosci. 2013;8(1):15-26.

48. Vago DR, Silbersweig DA. Self-awareness, self-regulation, and self-transcendence (S-ART): a framework for understanding the nurobiological mechanisms of mindfulness. Front Hum Neurosci. 2012; $6: 296$.

49. Freeman D, Garety PA, Kuipers E, Fowler D, Bebbington PE. A cognitive model of persecutory delusions. Br J Clin Psychol. 2002; 41(Pt 4):331-347.

50. Leykin Y, DeRubeis RJ. Allegiance in psychotherapy outcome research: separating association from bias. Clin Psych: Sci Practice. 2009;16(1):54-65.

51. Andreasen N, Carpenter W Jr, Kane JM, Lasser RA, Marder SR, Weinberger DR. Remission in schizophrenia: proposed criteria and rationale for consensus. Am J Psychiatry. 2005;162(3):441-449.
Neuropsychiatric Disease and Treatment

\section{Publish your work in this journal}

Neuropsychiatric Disease and Treatment is an international, peerreviewed journal of clinical therapeutics and pharmacology focusing on concise rapid reporting of clinical or pre-clinical studies on a range of neuropsychiatric and neurological disorders. This journal is indexed on PubMed Central, the 'PsycINFO' database and CAS,

\section{Dovepress}

and is the official journal of The International Neuropsychiatric Association (INA). The manuscript management system is completely online and includes a very quick and fair peer-review system, which is all easy to use. Visit http://www.dovepress.com/testimonials.php to read real quotes from published authors. 